\section{Case Report}

Korean J Transplant 2021;35:189-194 https://doi.org/10.4285/kjt.21.0010

\section{$\mathrm{KJT}$ \\ KOREAN JOURNAL OF TRANSPLANTATION}

\title{
Clinical sequence of an adult recipient undergone split liver transplantation using a right liver graft with erroneous deprivation of the middle hepatic vein trunk: a case report
}

\author{
Geunhyeok Yang, Shin Hwang, Chul-Soo Ahn, Tae-Yong Ha, Dong-Hwan Jung \\ Division of Hepatobiliary Surgery and Liver Transplantation, Department of Surgery, Asan Medical Center, \\ University of Ulsan College of Medicine, Seoul, Korea
}

Received May 18, 2021

Revised June 8, 2021

Accepted June 8, 2021

Corresponding author: Shin Hwang Department of Surgery, Asan Medical Center, University of Ulsan College of Medicine, 88 Olympic-ro 43-gil, Songpagu, Seoul 05505, Korea

Tel: +82-2-3010-3930

Fax: +82-2-3010-6701

E-mail: shwang@amc.seoul.kr

(c) The Korean Society for Transplantation This is an Open Access article distributed under the terms of the Creative Commons Attribution Non-Commercial License (http://creativecommons.org/licenses/ by-nc/4.0/) which permits unrestricted non-commercial use, distribution, and reproduction in any medium, provided the original work is properly cited.
The anatomy of middle hepatic vein (MHV) varies widely, and some individuals have aberrant MHV anatomy, thus there is risk of iatrogenic damage to graft MHV during liver splitting. We present the clinical sequences of an adult recipient who received a split right liver graft with erroneous deprivation of the MHV trunk. This is the case was a 58-year-old male patient with hepatitis B virus-associated liver cirrhosis who suffered from hepatic encephalopathy. The split right liver graft had a graft-to-recipient weight ratio of $2.1 \%$. Soon after graft reperfusion, large-sized hepatic venous congestion (HVC) appeared at the graft liver surface, indicating lack of MHV drainage. The amount of HVC was approximately $20 \%$ of the right liver graft mass at day 1 , which had gradually reduced on follow-up computed tomography (CT) scans. Although liver function recovered progressively, the patient remained bed-ridden because of pre-existing hypoxic brain damage. The patient passed away 4 years after transplantation because of pneumonia and multi-organ failure. The present case implies that there is some possibility of unrecognized damage to the graft MHV during liver splitting, suggesting the necessity of preoperative donor abdomen CT scan and preparation of intraoperative ultrasonography for easy evaluation of graft liver MHV anatomy.

Keywords: Middle hepatic vein; Donor shortage; Extended right liver graft; Hepatic venous congestion; Hepatic encephalopathy; Case report

\section{INTRODUCTION}

There has been a persistent shortage of deceased donor organs for liver transplantation (LT), and split liver transplantation (SLT) was developed to address this issue [1]. The proportion of SLTs was approximately $6 \%$ of all deceased donor LTs in the 2000s in the European Liver Transplant Registry [2,3], less than $1 \%$ of all LTs between 2002 and 2009 in the United States [4], and 3.5\% from 2005 to 2014 in Korea [5]. It has often been reported that the survival rate of SLT recipients does not differ significantly from whole liver transplant recipients [6,7]. Conventional SLTs involve dividing the deceased donor liver into a left lateral section (LLS) graft for a pediatric recipient and an extended right liver graft for an adult recipient. The usual LLS graft is actually an extended LLS graft with preservation of the middle hepatic vein (MHV) trunk at the right liver side and the retained left medial section parenchyma 


\section{HIGHLIGHTS}

- We present the clinical sequences of a 58-year-old adult recipient who received a split right liver graft with erroneous deprivation of the middle hepatic vein trunk.

- The present case implies that there is some possibility of unrecognized damage to the graft middle hepatic vein during liver splitting.

undergoes atrophies because of complete transection of the inflow vessels. The anatomy of the MHV trunk varies widely, and some individuals have aberrant MHV anatomy [8]. Thus, it is necessary to identify the running course of the MHV before and during liver splitting. We present the clinical sequences in a case of adult SLT recipient who received a right liver graft with erroneous deprivation of the MHV trunk.

\section{CASE REPORT}

The study protocol was approved by the Institutional Review Board at our institution (IRB No. 2021-0427), which waived the requirement for informed consent due to the retrospective nature of this study.

A 58-year-old male patient with hepatitis B virus-associated liver cirrhosis was admitted to our institution for hepatic encephalopathy. Because of portal hypertension secondary to advanced liver cirrhosis, the patient received endoscopic variceal ligation twice and transjugular intrahepatic portosystemic shunt (TIPS) twice (Fig. 1). Serum total bilirubin was $5.2 \mathrm{mg} / \mathrm{dL}$, prothrombin time international normalized ratio was 1.53 , and creatinine was normal, resulting in a model for end-stage liver disease (MELD) score of 17. Brain computed tomography (CT) showed diffuse brain swelling with poor differentiation between the grey matter and white matter junction involving bilateral cerebral hemispheres (Fig. 2A). Diffuse low-attenuation involving both the insular cortex and the temporal lobe suggested severe hepatic encephalopathy or hypoxic change. Because of altered mentality, the patient was intubated with ventilatory support. Inotropics were used to maintain vital signs. The patient was enrolled on the waiting list with the old Korean Network for Organ Sharing (KONOS) status 2A. After waiting for 8 days, the patient was selected for SLT.

The deceased donor was a 29-year-old male admitted to another hospital. In situ liver splitting was performed by the LLS graft-harvesting team. The split whole liver graft was harvested and divided at the back table at the donor hospital. The right liver graft weighed $1,450 \mathrm{~g}$, making a graft-to-recipient weight ratio (GRWR) of $2.1 \%$. A $1.5-\mathrm{cm}$ -
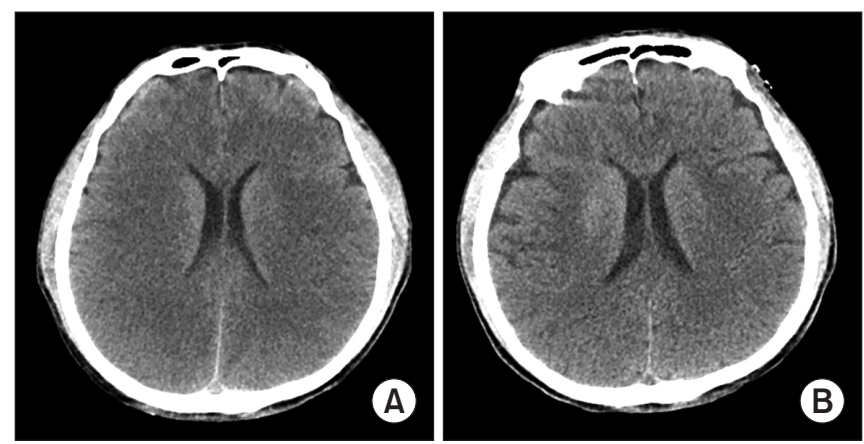

Fig. 2. Pretransplant brain computed tomography (CT) findings. (A) CT taken 2 days prior to transplantation shows diffuse brain swelling with poor differentiation at the grey matter and white matter junction involving bilateral cerebral hemispheres. (B) CT taken 3 days following transplantation shows resolution of brain swelling with improvement of brain perfusion.
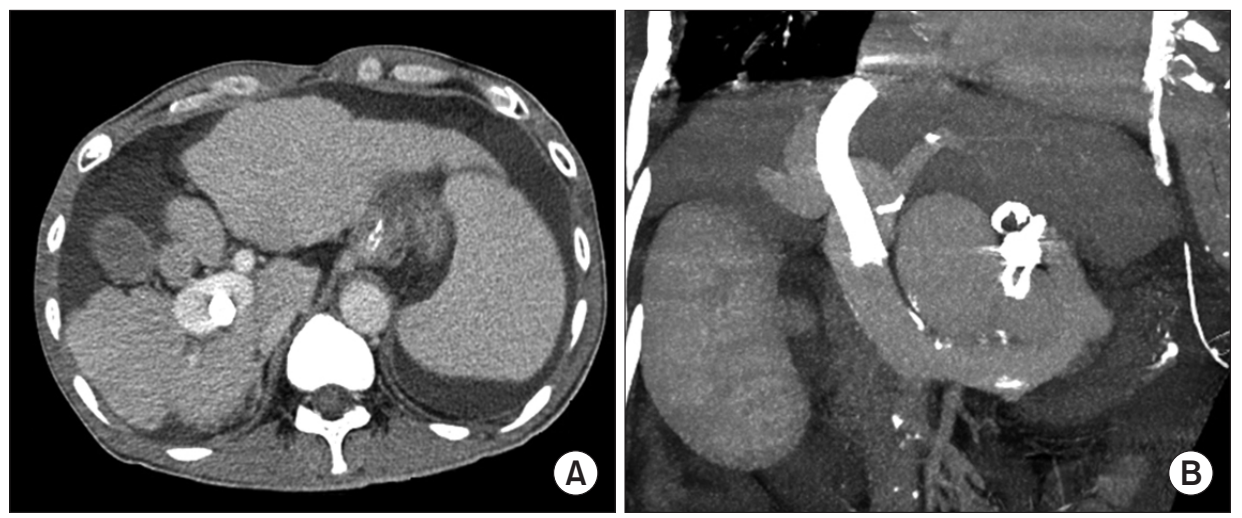

Fig. 1. Pretransplant computed tomography findings. (A) There is a cirrhotic liver with massive ascites. (B) Transjugular intrahepatic portosystemic shunt is located between the suprahepatic inferior vena cava and the main portal vein. 

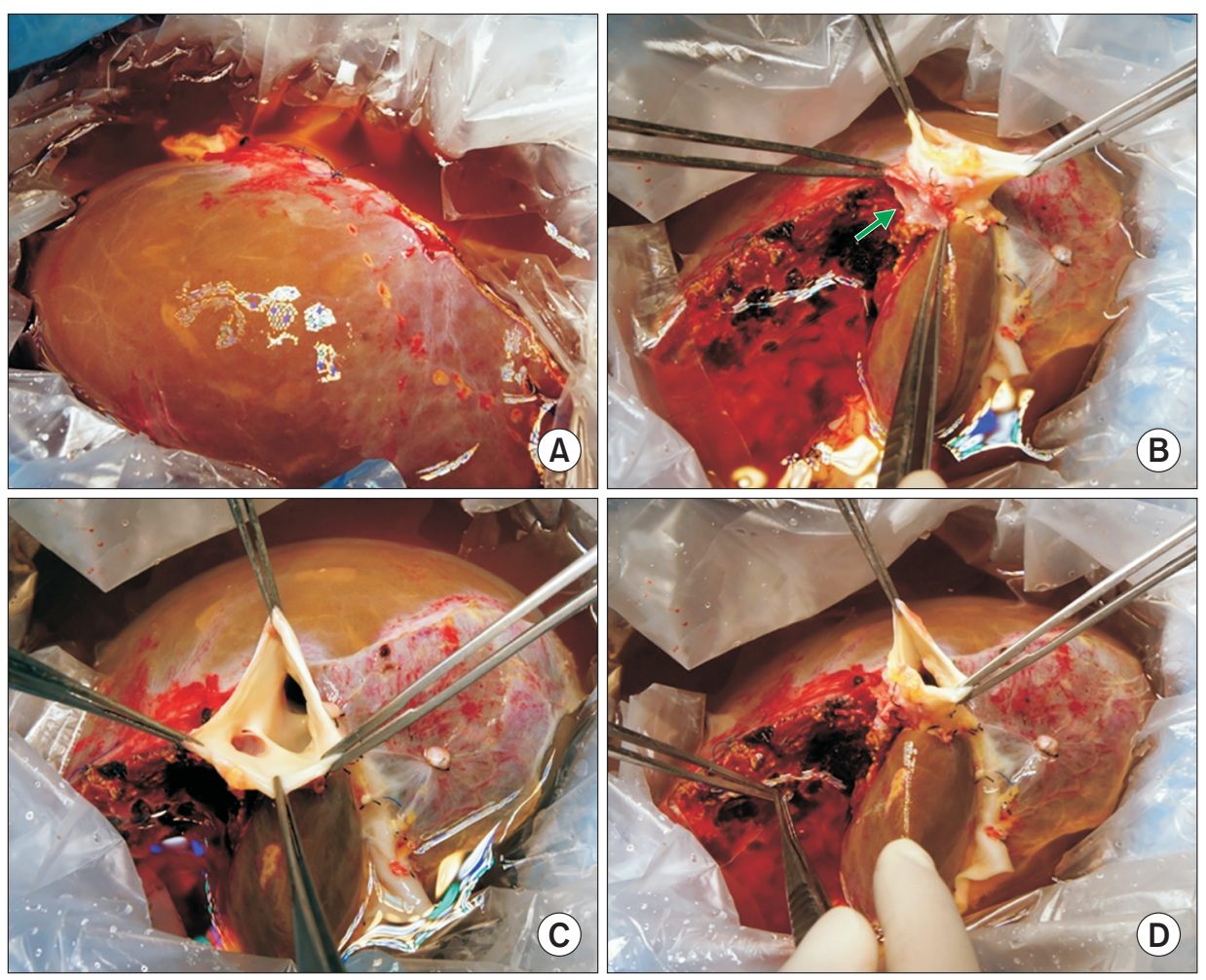

Fig. 3. Gross photographs of graft at bench work. (A) The hemihepatic discoloration line is marked at the liver surface. (B) A $1.5-\mathrm{cm}$ sized wall defect (arrow) is identified at the suprahepatic inferior vena cava (IVC) of the right liver graft. The wall defect corresponds to the graft hepatic vein orifice of the split left lateral section graft. (C) The internal lumens of the suprahepatic IVC are visible. (D) The wall defect at the suprahepatic IVC is closed with primary sutures.

sized wall defect was identified at the suprahepatic inferior vena cava (IVC) of the right liver graft, which was closed with primary sutures during bench work (Fig. 3).

Recipient hepatectomy was performed according to the standard procedures for deceased donor LT. The TIPS catheter was removed from the suprahepatic stump of the recipient IVC by pulling out of the transected TIPS wires. Active veno-venous bypass using a Bio-Pump (Medtronic, Minneapolis, MN, USA) was performed to divert the portal and infrahepatic IVC blood flow. The split right liver graft with the retrohepatic IVC was implanted according to the standard procedures for deceased donor whole LT. The graft IVC was interposed between the recipient's suprahepatic and infrahepatic IVC stumps and the graft portal vein was anastomosed to the intact recipient portal vein after resecting the TIPS catheter-inserted portion.

Soon after graft reperfusion with the portal blood flow, a large-sized hepatic venous congestion (HVC) appeared at the right anterior section of the right liver graft (Fig. 4). This HVC partially disappeared after hepatic arterial reperfusion. Duct-to-duct anastomosis with T-tube insertion was performed for biliary reconstruction. The explanted liver graft showed hepatitis B virus-associated mixed macronodular and micronodular cirrhosis (Fig. 5).

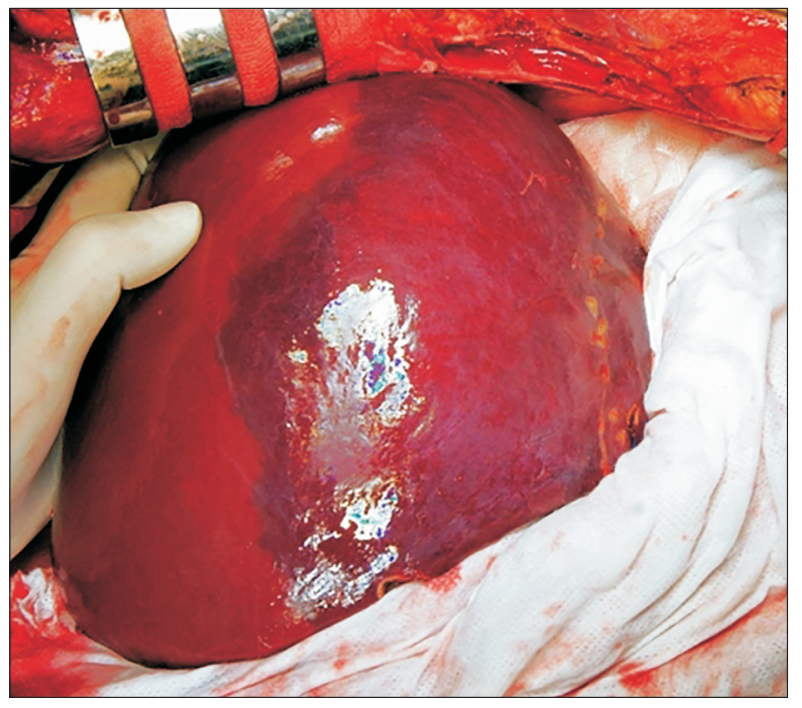

Fig. 4. Intraoperative photograph of the liver graft. A large-sized hepatic venous congestion occurred at the right anterior section of the right liver graft soon after graft reperfusion with the portal blood flow.

On posttransplant day 1 , serum aspartate transaminase and alanine transaminase raised to $3,561 \mathrm{IU} / \mathrm{L}$ and 2,980 IU/L, respectively, and liver CT showed a large-sized perfusion defect at the right anterior section of the liver 
graft (Fig. 6A). Follow-up brain CT at days 3 showed resolution of brain swelling with improvement of brain perfusion (Fig. 2B). On posttransplant day 7, the extent of perfusion defect decreased with the development of intrahepatic venous collaterals (Fig. 6B). HVC had nearly completely resolved at the 6-month $\mathrm{CT}$ (Fig. $6 \mathrm{C}$ ), and it had disappeared on the follow-up CT scans taken later (Fig. 6D).

Because hypoxic brain damage occurred prior to LT probably due to hepatic encephalopathy, the patient re-

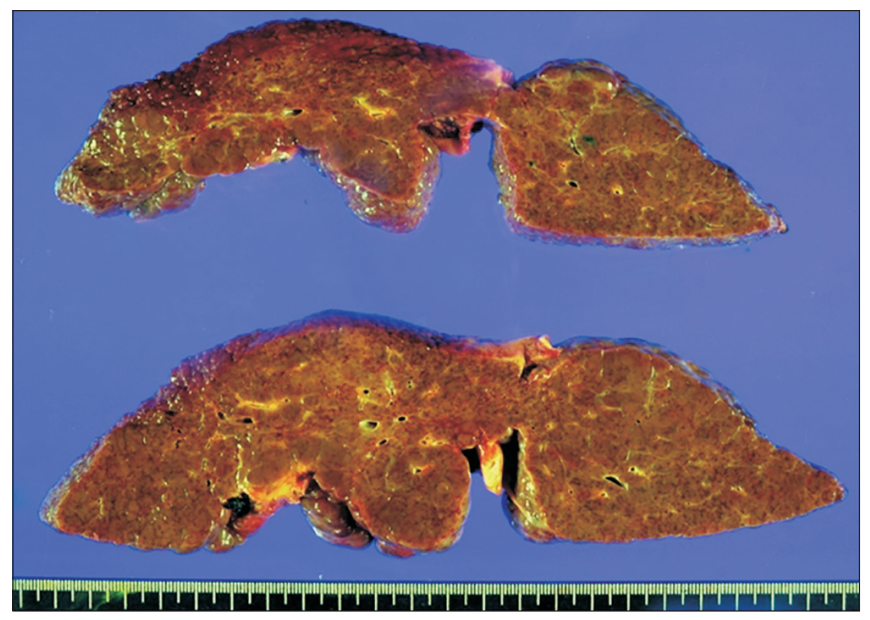

Fig. 5. Gross photograph of the explanted liver. Hepatitis B virus-associated mixed macronodular and micronodular cirrhosis is visible. mained bed-ridden state with a T-cannula at the tracheostomy and was fed through a nasogastric tube. The patient was discharged to a supportive care facility 3 months after the LT operation. At posttransplant 8 months, spontaneous subdural hemorrhage occurred, which was managed with supportive care. The patient was admitted several times because of gastrointestinal bleeding, ileus, pancytopenia, early chronic rejection, and other problems. The patient passed away 4 years after LT from pneumonia and multi-organ failure.

\section{DISCUSSION}

Since the first child-adult SLT was conducted in Korea in 1998 [9], the number of SLT cases has remained low, but has been gradually increasing because of progressive increase in the number of deceased donors and changes in the allocation policy for SLT $[10,11]$. With regard to adult recipients of split extended right liver grafts, posttransplant outcome is a matter of concern. In a multicenter study conducted in Korea, the risk factors for poorer patient survival outcomes in SLT recipients were GRWR $\leq 1.0$ and MELD score $>30$ [5].

We previously reported that 242 adult patients underwent a total of 256 deceased donor liver LT between June 2016 and November 2019 and SLT was performed in
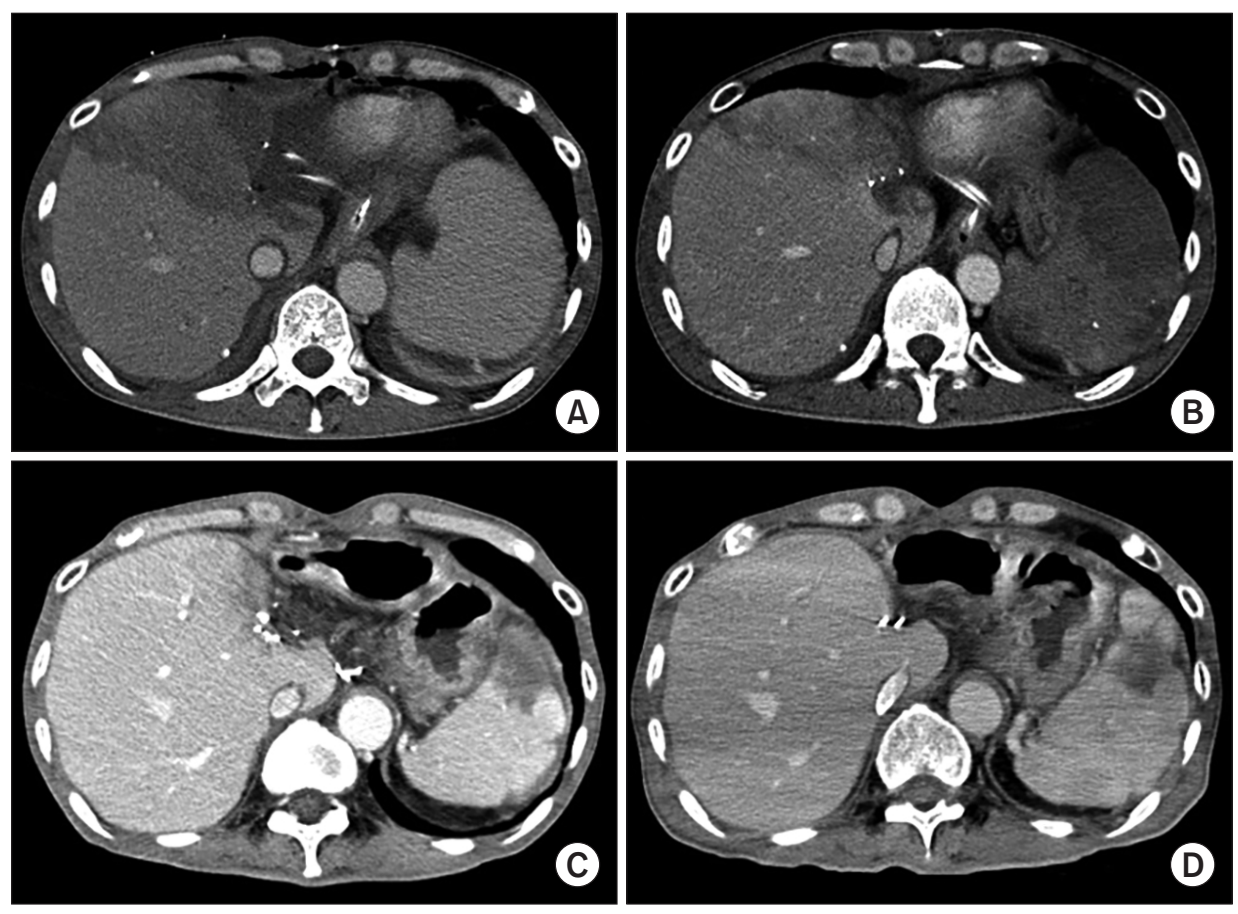

Fig. 6. Posttransplant follow-up computed tomography (CT) scans. (A) CT taken at day 1 shows a large-sized perfusion defect at the right anterior section of the liver graft. (B) CT taken at day 7 shows a reduction of the perfusion defect and the development of intrahepatic venous collaterals. (C) CT taken after 6 months shows nearly complete resolution of hepatic venous congestion-associated perfusion defect. (D) CT taken after 1 year shows disappearance of perfusion defect at the liver graft. 
seven patients $(2.9 \%)$. The mean age of SLT donors was $29.7 \pm 7.4$ years, and mean split right liver graft weight was $1228.6 \pm 149.7 \mathrm{~g}$ with mean GRWR of $1.97 \pm 0.39$. Of the seven SLT recipients, the MELD score-based KONOS status was one in status 1 , one in status 2 and five in status 3 . The graft and patient survival rates were comparable in the SLT and whole LT groups. Univariate analysis showed that pretransplant ventilator support and renal replacement therapy were significantly associated with patient survival, whereas KONOS status category and primary liver diseases were not. Multivariate analysis showed that pretransplant ventilator support was an independent risk factor for patient survival [7]. We concluded that that the outcome of adult SLT was comparable to that of whole LT, and we suggested that careful patient selection is essential to improve the outcomes of SLT.

The usual split right liver graft is an extended right liver graft because the retained left medial section (S4) parenchyma beyond the MHV trunk will undergo atrophies as a result of the transection of the inflow vessels. We previously reported the fates of retained left medial section parenchyma in 25 adult SLT recipients [12]. The mean donor age was $26.5 \pm 7.7$ years. The split extended right liver graft weight was $1,181.5 \pm 252.8 \mathrm{~g}$, which resulted in a mean GRWR of $1.98 \pm 0.44$. CT of the retained S4 parenchyma revealed small ischemic necrosis in 16 patients (64\%) and large ischemic necrosis in the remaining nine patients $(36 \%)$. The mean GRWR was $1.87 \pm 0.43$ in the nine patients with large ischemic necrosis and $2.10 \pm 0.44$ in the 15 cases with small ischemic necrosis $(P=0.28)$. The retained $S 4$ parenchyma showed gradual atrophy on follow-up imaging studies. The amount of S4 ischemic necrosis was not associated with graft or patient survival. A MELD score $>30$ and pretransplant ventilator support were associated with inferior outcomes.

The anatomy of the MHV varies widely, and some individuals have rare aberrant MHV anatomy. However, the detailed intrahepatic vascular anatomy of the donor liver has not been routinely evaluated in the majority of deceased donors. In practice, such anatomical assessment is not necessary for the usual whole LT. As a result, donor surgeons performing liver splitting usually select the parenchymal transection plane after observing the hemihepatic inflow occlusion-inducing discoloration and their own personal experience. If the donor liver does not have the usual anatomy of the MHV trunk running along the Cantlie line [8], the parenchymal transection plane for splitting should be corrected with intraoperative ultrasonography. However, in- traoperative ultrasonography is also not readily available in most situations involving deceased donor organ recovery. As a result, there is a potential risk of damage to the graft MHV during splitting into an extended right liver graft and an LLS graft, as shown in the present case. We presume that the graft MHV damage might be caused by some unusual aberrant MHV anatomy rather than lack of surgeons' experience. Special attention should be paid for splitting of the donor livers with relatively small-sized S4 parenchyma.

We have learned much about the adverse effects of MHV flow deprivation during living donor LT using a right liver graft. The liver graft in the present case was comparable to a living donor right liver graft without the MHV trunk. The HVC at the right anterior section might not be identified during donor splitting surgery because the hepatic arterial flow was maintained until cross-clamping of the donor aorta. In the present case, after graft reperfusion with portal blood flow, the ischemic congestion at the right anterior section was overtly manifested, as we have often observed during living donor LT. We could not recognize the extent of HVC before graft reperfusion. If we had recognized the possibility of MHV damage occurred during donor liver splitting, the extent of HVC would be assessed intraoperatively and the transected MHV branches would be reconstructed with a conduit vessel at the back table [13-15]. The MHV deprivation-associated HVC at the liver graft resolved slowly within a few weeks. We previously reported the mechanisms of resolving the HVC that develops after occlusion of the interposed MHV conduits. CT imaging analysis indicated that extrinsic compression of conduits, development of intrahepatic collaterals, and conduit shrinkage were the main mechanisms underlying late MHV conduit occlusion. The timing of MHV conduit occlusion was well correlated with that of neo-collateralization [14].

The amount of HVC was estimated to be approximately $20 \%$ of the right liver mass in the present case. However, this did not induce critically detrimental effects on graft liver function because the functioning graft liver mass excluding the HVC portion was large enough. If the split liver graft had been small-for-size, serious deterioration of the graft function would have occurred, as we have occasionally experienced in living donor LT. The disappointing clinical course after LT was primarily due to hepatic encephalopathy-associated brain damage. Massive HVC from graft MHV damage was not the main cause of poor posttransplant outcome because early allograft dysfunction was not so serious and graft function recovered progressively. We previously reported that pretransplant ventilator support is 
a risk factor for poor prognosis in adult SLT recipients [7].

In conclusion, our experience from the present case implies that there is some possibility of unrecognized damage to the graft MHV during liver splitting. Therefore, it is reasonable to take abdomen $\mathrm{CT}$ scan in deceased donors who are selected to be SLT donors and to prepare intraoperative ultrasonography for ease of use.

\section{ACKNOWLEDGMENTS}

\section{Conflict of Interest}

No potential conflict of interest relevant to this article was reported.

\section{Funding/Support}

This study was supported by research grant from the Korean Society for Transplantation (2021-00-03007-019).

\section{ORCID}

Geunhyeok Yang

Shin Hwang

Chul-Soo Ahn

Tae-Yong $\mathrm{Ha}$

Dong-Hwan Jung

https://orcid.org/0000-0002-8260-1525 https://orcid.org/0000-0002-9045-2531 https://orcid.org/0000-0002-3844-3646 https://orcid.org/0000-0001-9932-0212 https://orcid.org/0000-0001-5984-023X

\section{Author Contributions}

Conceptualization: SH. Data curation: CSA, TYH, DHJ. Formal analysis: GY, SH. Methodology: CSA, TYH, DHJ, SH. Project administration: $\mathrm{SH}$. Visualization: $\mathrm{SH}$. Writing-original draft: GY, SH. Writing-review \& editing: All authors.

\section{REFERENCES}

1. Broelsch CE, Emond JC, Whitington PF, Thistlethwaite JR, Baker AL, Lichtor JL. Application of reduced-size liver transplants as split grafts, auxiliary orthotopic grafts, and living related segmental transplants. Ann Surg 1990;212:368-75.

2. Renz JF, Yersiz H, Reichert PR, Hisatake GM, Farmer DG, Emond JC, et al. Split-liver transplantation: a review. Am J Transplant 2003;3:1323-35.

3. Adam R, McMaster P, O'Grady JG, Castaing D, Klempnauer $\mathrm{JL}$, Jamieson $\mathrm{N}$, et al. Evolution of liver transplantation in Europe: report of the European Liver
Transplant Registry. Liver Transpl 2003;9:1231-43.

4. Superina R. To split or not to split: that is the question. Liver Transpl 2012;18:389-90.

5. Chul Yoon K, Song S, Jwa EK, Lee S, Man Kim J, Kim OK, et al. Survival outcomes in split compared with whole liver transplantation. Liver Transpl 2018;24:1411-24.

6. Cardillo M, De Fazio N, Pedotti P, De Feo T, Fassati LR, Mazzaferro V, et al. Split and whole liver transplantation outcomes: a comparative cohort study. Liver Transpl 2006;12:402-10.

7. Park GC, Hwang S, Song GW, Jung DH, Ha TY, Ahn CS, et al. Prognosis of split liver transplantation compared with whole liver transplantation in adult patients: single-center results under the Korean MELD score-based allocation policy. J Korean Med Sci 2020;35:e304.

8. Hwang S, Lee SG, Choi ST, Moon DB, Ha TY, Lee YJ, et al. Hepatic vein anatomy of the medial segment for living donor liver transplantation using extended right lobe graft. Liver Transpl 2005;11:449-55.

9. Suh KS, Lee KW, Koh YT, Roh HR, Chung JK, Minn KW, et al. First successful in situ split-liver transplantation in Korea. Transplant Proc 2000;32:2140.

10. Cho WH. Organ donation in Korea in 2018 and an introduction of the Korea national organ donation system. Korean J Transplant 2019;33:83-9.

11. Ha HS, Hong JJ, Kim IO, Lee SR, Lee AY, Ha TY, et al. Deceased donor liver transplantation under the Korean model for end-stage liver disease score-based liver allocation system: 2-year allocation results at a high-volume transplantation center. Korean J Transplant 2019;33:112-7.

12. Chung YK, Hwang S, Ahn CS, Kim KH, Moon DB, Ha $T Y$, et al. Fates of retained hepatic segment IV and its prognostic impact in adult split liver transplantation using an extended right liver graft. Ann Surg Treat Res 2021;101:1-12.

13. Hwang S, Lee SG, Park KM, Kim KH, Ahn CS, Lee YJ, et al. Hepatic venous congestion in living donor liver transplantation: preoperative quantitative prediction and follow-up using computed tomography. Liver Transpl 2004;10:763-70.

14. Kirchner VA, Hwang S, Song GW, Ahn CS, Moon DB, $\mathrm{Kim} \mathrm{KH}$, et al. Resolution of hepatic venous congestion following gradual occlusion of middle hepatic vein interposition graft in living donor liver transplantation. Ann Transplant 2016;21:619-25.

15. Hwang S, Ha TY, Ahn CS, Moon DB, Kim KH, Song GW, et al. Standardized surgical techniques for adult living donor liver transplantation using a modified right lobe graft: a video presentation from bench to reperfusion. Korean J Hepatobiliary Pancreat Surg 2016;20:97-101. 